\title{
Intoxicação experimental por cebola, Allium cepa (Liliaceae), em gatos ${ }^{1}$
}

\author{
Rafael A. Fighera ${ }^{2}$, Tatiana M. Souza² ${ }^{\text {, Ingeborg Langohr }{ }^{2} \text { e Claudio S. L. Barros }}{ }^{2}$ \\ ABSTRACT.- Fighera R.A., Souza T.M., Langohr I. \& Barros C.S.L. 2002. [Experimental onion \\ Allium cepa (Liliaceae) poisoning in cats.] Intoxicação experimental por cebola, Allium \\ cepa (Liliaceae), em gatos. Pesquisa Veterinária Brasileira 22(2):79-84. Depto Patologia, \\ Universidade Federal de Santa Maria, 97105-900 Santa Maria, Rio Grande do Sul, Brazil. E- \\ mail: anemiaveterinaria@bol.com.br \\ Onion poisoning is reported worlwide in several animal species. The toxic principle (n- \\ propyl dissulfide) present in onions causes the transformation of hemoglobin in \\ methemoglobin. In order to study the laboratory, gross and histopathological findings in \\ onion poisoning in cats, five 4-month-old cats were fed a single dose of $10 \mathrm{~g} / \mathrm{kg}$ of dried-onion \\ each. Another cat of the same age did not receive the onion meal and served as control. All \\ five cats developed clinical signs of the toxicosis; one of them died within 24 hours of the \\ ingestion of the onion meal. Clinical signs included apathy, tachycardia, tachypnea, and \\ cyanosis. Laboratory findings included hemolytic anemia associated with Heinz bodies and \\ methemoglobinemia. Main necropsy findings were splenomegaly and brown discoloration of \\ blood. Histopathological findings included splenic and hepatic hemosiderosis and multifocal \\ extramedullary hematopoiesis.
}

INDEX TERMS: Poisonous plants, Allium cepa, onion, hemolytic anemia, methemoglobinemia, Heinz bodies, diseases of cats, pathology.

RESUMO.- A intoxicação por cebola é relatada em várias espécies animais em muitas partes do mundo. $O$ princípio tóxico (n-propil dissulfito) presente na cebola causa a transformação da hemoglobina em metemoglobina. Para estudar os achados laboratoriais, de necropsia e histopatológicos da intoxicação por cebola em gatos, cinco gatos de quatro meses de idade receberam cada um uma dose única de $10 \mathrm{~g} / \mathrm{kg}$ de cebola desidratada por via oral. Um outro gato de mesma idade não recebeu a refeição com cebola e serviu como controle. Todos os cinco gatos desenvolveram sinais clínicos da toxicose; um deles morreu dentro de $\mathbf{2 4}$ horas após a ingestão da cebola. Os sinais clínicos incluíram apatia, taquicardia, taquipnéia e cianose. Os achados laboratoriais se caracterizavam por anemia hemolítica associada a corpúsculos de Heinz e metemoglobinemia. Os principais achados de

\footnotetext{
${ }^{1}$ Aceito para publicação em 17 de maio de 2002.

Trabalho financiado pelo Programa de Apoio a Núcleos de Excelência (PRONEX), Proc. $\mathrm{n}^{\mathrm{o}} 7697102600$.

${ }^{2}$ Departamento de Patologia, Universidade Federal de Santa Maria, 97105900, Santa Maria, RS, Brasil.
}

necropsia foram esplenomegalia e sangue de cor marrom. Os achados histopatológicos foram hemossiderose e hematopoese extramedular no baço e fígado.

TERMOS DE INDEXAÇÃO: Intoxicação por plantas, Allium cepa, cebola, anemia hemolítica, metemoglobinemia, corpúsculos de Heinz, doenças de gatos, patologia.

\section{INTRODUÇÃO}

A intoxicação por cebola comum (Allium cepa) e cebola silvestre (Allium validum) sob condições naturais é descrita em cães (Gruhzit 1931), gatos (Kaplan 1995, Robertson 1998, Fighera 2001), equiinos (Thorp \& Harshfield 1939), ovinos (Van Kampen et al. 1970) e bovinos (Koger 1956) em todo o mundo, sendo considerada por muitos autores como uma das primeiras toxicoses diagnosticadas em grandes animais no continente americano (Searcy 1998). Sabe-se que a intoxicação ocorre ainda hoje em cavalos e ruminantes que invadem lavouras ou que têm acesso a locais onde exista cebola silvestre (Hutchinson 1977). Em cães, a intoxicação ocorre pelo uso de sobras da alimentação humana na dieta do animal, independentemente da cebola estar crua, cozida ou desidratada (Farkas \& Farkas 1974). Em gatos, a intoxicação natural é as- 
sociada ao consumo de comida enlatada para bebês, um alimento rotineiramente utilizado para gatos enfermos, devido a sua alta palatabilidade (Kaplan 1995). Além disso, anemia hemolítica com formação de corpúsculos de Heinz é relatada em vários gatos que ingeriram sopa de cebola ou extrato de cebola crua (Robertson 1998). Relata-se ainda a reprodução experimental dessa intoxicação em gatos submetidos por dois meses a alimentação exclusivamente a base de alimento enlatado para bebês (Robertson 1998).

A intoxicação espontânea por cebola está associada a duas manifestações clínico-patológicas. Uma condição aguda, caracterizada por metemoglobinemia grave que causa cianose e morte (Harvey \& Rackear 1985), e outra, um pouco mais crônica, decorrente de anemia hemolítica com formação de corpúsculos de Heinz (Jain 1993). Os animais que ingerem grande quantidade da planta de uma única vez desenvolvem metemoglobinemia, tornando-se apáticos, hipotérmicos e cianóticos (Weiser 1992). Animais que não morrem ou que ingerem pequenas quantidades da planta por vários dias desencadeiam um quadro hemolítico, tornando-se também apáticos e apresentando mucosas hipocoradas e metemoglobinúria (Jain 1986).

A reprodução experimental da intoxicação por cebola em gatos tem como objetivo descrever com detalhes as alterações clínicas, laboratoriais e patológicas dessa enfermidade e validar essa espécie como modelo experimental para o estudo dessa toxicose. A escolha dos felinos para a realização do experimento justifica-se por esses animais serem mais susceptíveis em desenvolver anemia cujo mecanismo patogenético envolva a formação de corpúsculos de Heinz (Boothe 1990, Weiser 1994).

\section{MATERIAL E MÉTODOS}

No experimento foram usados seis gatos, machos e fêmeas, sem raça definida, com aproximadamente quatro meses de idade. Antes do início do experimento, os animais foram identificados com números de 1 a 6 e tratados com anti-helmíntico de amplo espectro. Com a finalidade de proporcionar adaptação ao novo ambiente, os gatos foram mantidos por quinze dias em gaiolas individuais recebendo ração comercial de boa qualidade e água à vontade. Um dia antes da administração da cebola, foi realizado hemograma e exame comum de urina em cada um dos seis gatos. A planta foi administrada a cinco gatos, numa dose única de $10 \mathrm{~g} / \mathrm{kg}$, em forma de cebola desidratada misturada a $50 \mathrm{~g} / \mathrm{kg}$ de ração úmida de boa qualidade. 0 gato 6 recebeu apenas $50 \mathrm{~g} / \mathrm{kg}$ de ração úmida e serviu de controle. A mistura da planta com a ração teve como objetivo facilitar o consumo, pois a cebola desidratada é pouco palatável para felinos. Os animais foram observados clinicamente de três em três horas no primeiro dia após a administração da cebola e de oito em oito horas nos dias subsequientes. No exame clínico eram avaliados a frequiência cardíaca, freqüência respiratória, temperatura, coloração das mucosas e estado geral.

Todos os animais, exceto o gato 4, que morreu no primeiro dia após a administração da planta, foram submetidos a um novo hemograma no sétimo dia pós-administração. Nessas amostras de sangue foram também realizadas a pesquisa de corpúsculos de Heinz e a determinação da metemoglobina sérica. Para a realização do mielograma, todos os gatos foram anestesiados com uma combinação de cloridrato de quetamina $(20 \mathrm{mg} / \mathrm{kg})$ e cloridrato de xilazina ( $2 \mathrm{mg} / \mathrm{kg}$ ) e foi feita a punção aspirativa por agulha fina (PAAF) da medula óssea da crista ilíaca (Harvey 2001). A urina foi coletada por cistocentese. Ao final do experimento, os cinco gatos restantes foram submetidos à eutanásia com injeção intracardíaca de cloreto de potássio $(500 \mathrm{mg} / \mathrm{kg})$. Todos os seis gatos foram necropsiados.

Os hemogramas foram feitos manualmente conforme técnicas convencionais (Vallada 1999), constituindo-se de 1) contagem de eritrócitos e leucócitos em câmara de Neubauer utilizando-se respectivamente líquido de Hayen e líquido de Turk como diluentes; 2) determinação do hematócrito por centrifugação de microcapilares por cinco minutos a $7.000 \mathrm{RPM}$; 3) determinação do volume corpuscular médio (VCM) por cálculo; e 4) análise de esfregaços corados por panótico rápido para a diferenciação celular e pesquisa de alterações morfológicas nas células. A determinação de hemoglobina pelo método da cianometemoglobina utilizando reagentes comerciais e conseqüientemente a obtenção da concentração de hemoglobina corpuscular média ( $\mathrm{CHCM}$ ) só foi possível no primeiro dia, porque após a intoxicação, os altos níveis de metemoglobina inviabilizam a determinação de hemoglobina por qualquer técnica (Fighera 2001).

A pesquisa dos corpúsculos de Heinz foi realizada pela técnica da coloração supravital (Naoum 1997), através de solução de azul cresil brilhante. A metemoglobina sérica foi determinada por espectrofotometria a 630 nanômetros, após ensaios colorimétricos com reagentes específicos (Naoum 1997). Para realização dos hemogramas, para a pesquisa dos corpúsculos de Heinz e para a determinação da metemoglobina, cinco $\mathrm{ml}$ de sangue foram coletados da veia jugular com agulhas $25 \times 7 \mathrm{~mm}$ e seringas plásticas. Esse sangue foi acondicionado em tubos de ensaio com uma gota de anticoagulante etilenodiaminotetracetato de sódio (EDTA) a $10 \%$.

Cinco mililitros de urina foram coletados por cistocentese e acondicionados em frascos âmbar. 0 exame comum de urina foi realizado por técnicas tradicionais, avaliando-se os aspectos físicos, químicos e o sedimento (Navarro 1996). Para a avaliação química foram utilizadas fitas reagentes comerciais.

A obtenção da medula óssea para análise citológica (mielograma) foi realizada através de técnicas rotineiras de PAAF (Harvey 2001), usando para isso agulhas especiais e seringas plásticas contendo uma gota de EDTA a $10 \%$. Com o material obtido confeccionaram-se esfregaços que foram corados com panótico rápido. Fragmentos de fígado e baço colhidos na necropsia foram fixados em formol comercial a $10 \%$, processados rotineiramente para histopatologia e corados pela hematoxilina-eosina $(\mathrm{HE})$.

\section{RESULTADOS}

\section{Sinais clínicos}

Os achados clínicos encontrados nos gatos que ingeriram a cebola desidratada podem ser divididos de acordo com as duas formas clínicas descritas para a doença. Após nove horas do consumo, todos os gatos estavam acentuadamente apáticos, taquicárdicos, taquipnéicos, com as mucosas azuladas e normotérmicos. Esse quadro se manteve por aproximadamente 48 horas, com exceção do Gato 4 que morreu após doze horas da ingestão da cebola, em coma e sob severa hipotermia $\left(34,5^{\circ} \mathrm{C}\right)$. A partir do terceiro dia da ingestão da cebola os gatos já apresentavam as mucosas pálidas e, por volta do quinto dia, a taquicardia e a taquipnéia reapareceram, porém com menor intensidade. Nesse mesmo dia, era possível notar que os Gatos 1 e 3, apresentavam urina escura. Esse parâmetro não pôde ser avaliado nos outros animais. 

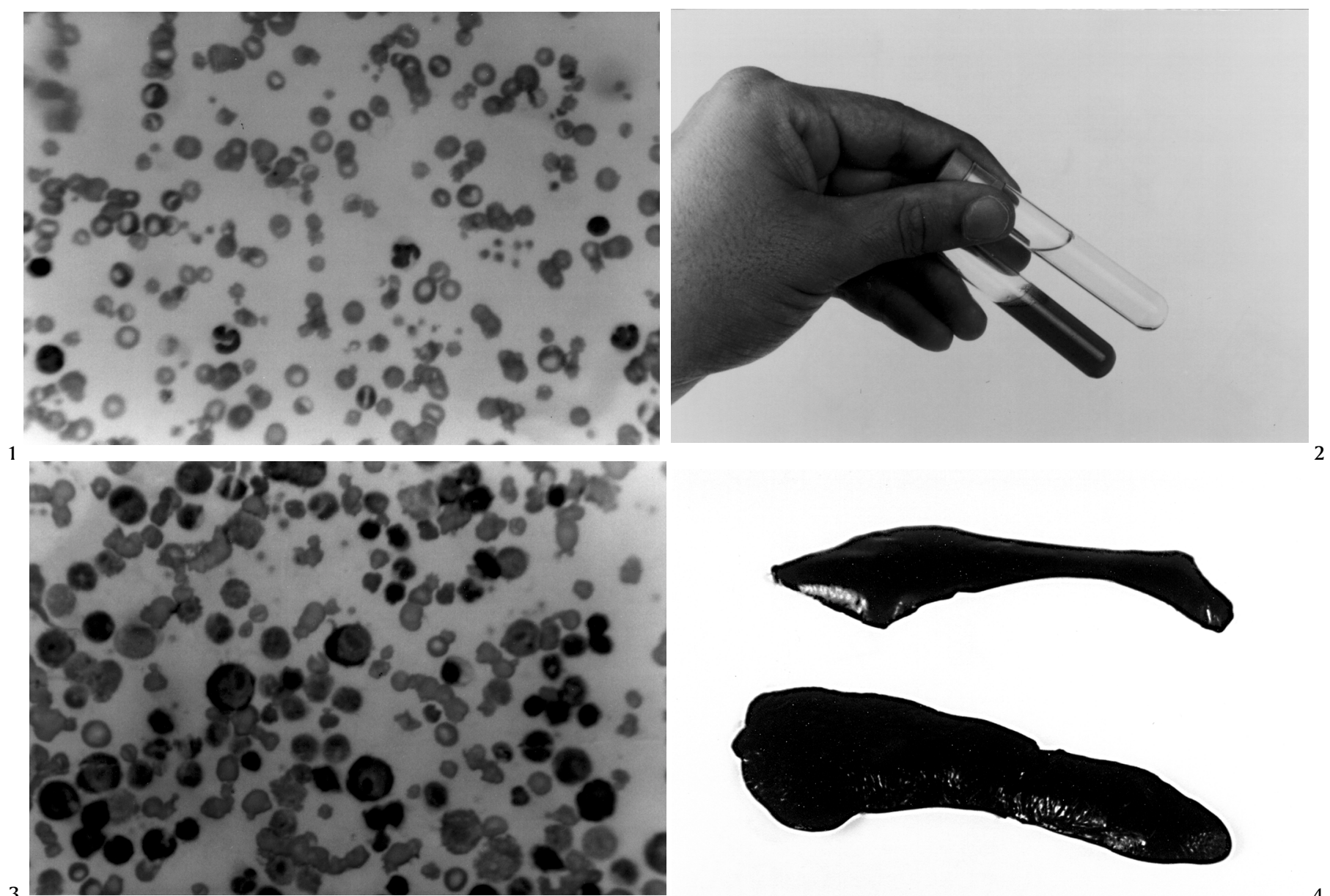

Fig. 1. Esfregaço sangüíneo do Gato 2 intoxicado por cebola (Allium cepa). Há grande quantidade de eritrócitos com corpúsculos de Heinz.

Fig. 3. Acentuada eritrogênese pode ser observada no esfregaço da medula óssea do Gato 3 intoxicado por cebola (Allium cepa).

Os sinais clínicos permaneceram constantes até o momento da eutanásia.

\section{Achados laboratoriais}

$\mathrm{O}$ hemograma de quatro gatos que consumiram a cebola desidratada demonstra uma anemia macrocítica hipocrômica aguda grave associada a alterações morfológicas do tecido eritróide compatíveis com intensa regeneração. Essas alterações foram policromasia, anisocitose, metarrubricitemia (normoblastemia) e presença de corpúsculos de Howell-Jolly. $\mathrm{O}$ plasma após centrifugação era marrom. Na análise do esfregaço era possível, mesmo na coloração de rotina, evidenciarem-se muitos eritrócitos com corpúsculos de Heinz (Fig. 1). Além disso, alguns excentrócitos podiam ser observados. Em relação ao leucograma, todos os animais apresentavam altas contagens de leucócitos, fenômeno que, quando associado a metarrubricitemia, é conhecido como reação

leucoeritroblástica. Os resultados individuais podem ser vistos no Quadro 1.

A pesquisa dos corpúsculos de Heinz foi positiva em quatro dos cinco gatos testados e a determinação dos níveis séricos de metemoglobina demonstrou um aumento variável nos gatos afetados, porém todos os valores estavam bem acima dos considerados fisiológicos para a espécie. A urinálise realizada no sétimo dia após a ingestão de cebola demonstrou urina escura (Fig. 2), levemente positiva para sangue oculto. Esses dados individuais estão expressos no Quadro 2.

No mielograma, uma eritrogênese acentuada foi observada nos gatos que consumiram a planta (Fig. 3), comprovada pela inversão na relação mielóide:eritróide e pela grande quantidade de precursores eritróides em atividade mitótica na medula óssea. Os resultados individuais dos mielogramas estão no Quadro 3. 
Quadro 1. Hemogramas dos gatos intoxicados experimentalmente por cebola, Allium cepa (Liliaceae)

\begin{tabular}{|c|c|c|c|c|c|c|c|c|c|c|}
\hline \multirow{2}{*}{$\begin{array}{l}\text { Gato } \\
\text { Dia }\end{array}$} & \multicolumn{2}{|c|}{1} & \multicolumn{2}{|c|}{2} & \multicolumn{2}{|c|}{3} & \multicolumn{2}{|c|}{5} & \multicolumn{2}{|c|}{ Controle } \\
\hline & 0 & 7 & 0 & 7 & 0 & 7 & 0 & 7 & 0 & 7 \\
\hline Eritrócitos $\left(\mathrm{x} 10^{6} / \mathrm{mm}^{3}\right)$ & 5,3 & 2,5 & 5,0 & 2,2 & 6,9 & 3,0 & 5,0 & 3,2 & 5,6 & 6,0 \\
\hline Hemoglobina $(\mathrm{g} / \mathrm{dl})$ & 10,0 & $\mathrm{NR}^{\mathrm{a}}$ & 9,4 & NR & 11,1 & NR & 8,4 & NR & 9,8 & 10,4 \\
\hline Hematócrito (\%) & 31,0 & 17,0 & 28,0 & 18,0 & 37,0 & 21,0 & 28,0 & 22,0 & 32,0 & 33,0 \\
\hline $\operatorname{VCM}(\mathrm{fl})$ & 58,5 & 68,0 & 56,0 & 81,8 & 53,6 & 70,0 & 56,0 & 68,8 & 57,1 & 55,0 \\
\hline CHCM (\%) & 32,2 & NR & 33,6 & NR & 30,0 & NR & 30,0 & NR & 30,6 & 31,5 \\
\hline Leucócitos (/mm³) & 10.500 & 57.700 & 15.500 & 37.900 & 11.000 & 72.500 & 12.300 & 142.200 & 9.500 & 8.900 \\
\hline Neutrófilos (\%) & 77 & 64 & 65 & 80 & 53 & 81 & 69 & 80 & 76 & 68 \\
\hline Neutrófilos $\left(/ \mathrm{mm}^{3}\right)$ & 8.085 & 36.928 & 10.075 & 30.320 & 5.830 & 58.725 & 8.487 & 113.760 & 7.220 & 6.052 \\
\hline Bastonetes (\%) & 1 & 0 & 1 & 0 & 0 & 725 & 2 & 6 & 1 & 0 \\
\hline Bastonetes $\left(/ \mathrm{mm}^{3}\right)$ & 105 & 0 & 155 & 0 & 0 & 1 & 246 & 8.532 & 95 & 0 \\
\hline Linfócitos (\%) & 20 & 29 & 23 & 18 & 35 & 12 & 19 & 10 & 19 & 24 \\
\hline Linfócitos $\left(/ \mathrm{mm}^{3}\right)$ & 2.100 & 16.733 & 3.565 & 6.822 & 3.850 & 8.700 & 2.337 & 14.220 & 1.805 & 2.136 \\
\hline Monócitos (\%) & 1 & 4 & 7 & 0 & 4 & 4 & 2 & 2 & 2 & 3 \\
\hline Monócitos (/mm³) & 105 & 2.308 & 1.085 & 0 & 440 & 2.900 & 246 & 2.844 & 190 & 267 \\
\hline Eosinófilos (\%) & 1 & 3 & 4 & 2 & 8 & 2 & 7 & 1 & 2 & 4 \\
\hline Eosinófilos $\left(/ \mathrm{mm}^{3}\right)$ & 105 & 1.731 & 620 & 758 & 880 & 1.450 & 861 & 1.422 & 190 & 356 \\
\hline Basófilos (\%) & 0 & 0 & 0 & 0 & 0 & 0 & 1 & 1 & 0 & 1 \\
\hline Basófilos (/mm³) & 0 & 0 & 0 & 0 & 0 & 0 & 123 & 1.422 & 0 & 89 \\
\hline Policromasia & $+^{\mathrm{b}}$ & +++ & + & +++ & - & +++ & - & ++ & - & - \\
\hline Anisoscitose & + & +++ & + & +++ & + & +++ & + & ++ & + & + \\
\hline Howell-Jolly & - & ++ & - & +++ & - & ++ & - & ++ & - & - \\
\hline Metarrubrícitos ${ }^{\mathrm{c}}$ & 0 & 18 & 0 & 4 & 0 & 2 & 0 & 3 & 0 & 0 \\
\hline Excentrócitos & - & + & - & ++ & - & + & - & + & - & - \\
\hline
\end{tabular}

Quadro 2. Pesquisa de corpúsculos de Heinz, determinação da metemoglobina e análise química da urina dos gatos intoxicados experimentalmente por cebola, Allium cepa (Liliaceae)

\begin{tabular}{lccccc}
\hline Gato & 1 & 2 & 3 & 5 & $\mathrm{C}^{\mathrm{a}}$ \\
\hline Corpúsculos de Heinz & $++^{\mathrm{b}}$ & +++ & +++ & +++ & - \\
Metemoglobina (\%) & 25,0 & 32,0 & 16,0 & 9,0 & 2,0 \\
Sangue oculto na urina & + & + & + & + & -
\end{tabular}

a Controle.

b - Negativo, + leve, ++ moderado, +++ acentuado.

\section{Achados de necropsia e histopatologia}

$\mathrm{Na}$ necropsia, todos os animais que ingeriram a planta apresentavam esplenomegalia moderada ou acentuada (Fig. 4) e o sangue era marrom. Ao exame histológico evidenciavam-se hemossiderose e focos de hematopoese extramedular no fígado e no baço. No gato controle havia também alguns focos de hematopoese no baço. Necrose centrolobular leve pôde ser vista no gato 3 .

\section{DISCUSSÃO}

A administração de $10 \mathrm{~g} / \mathrm{kg}$ de cebola desidratada causou intoxicação em todos os gatos usados nesse experimento, confirmando assim que essa planta é tóxica para felinos domésticos e que esses podem servir como modelo experimental para o estudo da intoxicação. A intoxicação por cebola foi reproduzida experimentalmente em gatos submetidos por dois meses a alimentação exclusivamente a base de alimento enlatado para bebês (Robertson 1998). Esses gatos foram separados em 5 grupos que receberam doses diferentes de cebola. Os dados obtidos demonstraram que a dose mínima para causar a intoxicação em gatos foi de 2,5\% de pó de cebola misturado ao alimento enlatado.

Neste experimento, confirmando o que é descrito na literatura, dois quadros clínicos distintos foram observados após o consumo da cebola. Um agudo que podia ser visto em apenas nove horas após a ingestão da cebola e outro um pouco mais crônico, diagnosticado no terceiro dia após a ingestão (Harvey \& Rackear 1985, Jain 1993).

Os sinais clínicos na forma aguda foram semelhantes aos descritos na literatura, ou seja, apatia, taquicardia, taquipnéia e cianose (Harvey \& Rackear 1985). Esse quadro reflete os altos níveis de metemoglobina formada por um processo de oxidação da hemoglobina, o que leva a uma deficiência no transporte de oxigênio para o tecido. Isso resulta na anóxia (Williams 1941, Harvey 1989) que no caso do gato 4, desse experimento, culminou em morte, precedida por coma e hipotermia grave. O mecanismo para formação de metemoglobina não está totalmente esclarecido, mas sabese que a cebola possui uma substância conhecida por n-propil dissulfito, um oxidante que, se acredita inibe enzimas das vias do metabolismo energético, que são a glicólise anaeróbica ou via Embden Meyerhof e a derivação da hexose monofosfato ou via pentose fosfato (Jain 1986). A glicólise anaeróbica além de ser a principal fonte de ATP do eritrócito, tem como fun- 
Quadro 3. Mielograma dos gatos intoxicados experimentalmente por cebola, Allium cepa (Liliaceae)

\begin{tabular}{|c|c|c|c|c|c|}
\hline Gato & $\begin{array}{c}\text { Valores } \\
\text { normais }\end{array}$ & 1 & 2 & 3 & 5 \\
\hline Rubriblastos & $0,17( \pm 0,29)$ & 0,6 & 1,2 & 1,8 & 2,0 \\
\hline Pré-rubrícitos & $1,00( \pm 0,54)$ & 2,2 & 2,2 & 0,8 & 1,8 \\
\hline Rubrícitos basofílicos & $4,02( \pm 1,56)$ & 18,2 & 17,8 & 11,2 & 3,0 \\
\hline Rubrícitos policromáticos & $17,57( \pm 4,48)$ & 3,4 & 3,4 & 1,4 & 2,2 \\
\hline Metarrubrícitos & $5,54( \pm 3,14)$ & 36,6 & 36,6 & 52,6 & 38,0 \\
\hline Rubrícitos em mitose & $0,43( \pm 0,24)$ & 0,2 & 0,2 & 0,4 & 0,4 \\
\hline Total (eritróide) & $28,74( \pm 4,64)$ & 61,2 & 61,4 & 68,2 & 47,4 \\
\hline Mieloblastos & $0,08( \pm 0,16)$ & 0 & 0 & 2,0 & 10,6 \\
\hline Promielócitos & $1,74( \pm 1,04)$ & 0 & 1,0 & 1,2 & 10,6 \\
\hline Mielócitos neutrofílicos & $4,31( \pm 2,49)$ & 1,6 & 1,8 & 2,0 & 10,6 \\
\hline Mielócitos eosinofílicos & $0,60( \pm 0,42)$ & 0,4 & 1,0 & 1,4 & 2,0 \\
\hline Mielócitos basofílicos & $0,11( \pm 0,11)$ & 0,2 & 0 & 0,2 & 0,2 \\
\hline Metamielócitos neutrofílicos & $10,06( \pm 3,20)$ & 3,0 & 3,0 & 2,2 & 3,0 \\
\hline Metamielócitos eosinofílicos & $0,54( \pm 0,39)$ & 0 & 0 & 1,0 & 1,2 \\
\hline Metamielócitos basofílicos & $0,03( \pm 0,07)$ & 0,2 & 0 & 0 & 0,4 \\
\hline Bastonetes neutrofílicos & $14,4( \pm 1,30)$ & 6,8 & 6,6 & 5,6 & 5,0 \\
\hline Bastonetes eosinofílicos & $0,49( \pm 0,40)$ & 1,2 & 1,0 & 0,8 & 2,4 \\
\hline Bastonetes basofilicos & 0 & 0 & 0 & 0 & 0,2 \\
\hline Neutrófilos segmentados & $12,86( \pm 4,85)$ & 17,4 & 16,8 & 9,2 & 1,4 \\
\hline Eosinófilos segmentados & $0,60( \pm 0,20)$ & 1,2 & 1,2 & 2,2 & 0 \\
\hline Basófilos segmentados & 0 & 0 & 0 & 0,2 & 0,4 \\
\hline Total (mielóide) & $45,86( \pm 3,78)$ & 32,0 & 32,4 & 28,0 & 48,2 \\
\hline Relação mielóide:eritróide & $1,63: 1( \pm 0,35)$ & $0,52: 1$ & 0,53:1 & $0,41: 1$ & $1,02: 1$ \\
\hline Hematogôneas & $0,83( \pm 0,75)$ & 0,2 & 0,2 & 0,4 & 0,4 \\
\hline Linfócitos & $16,13( \pm 2,92)$ & 2,8 & 4,8 & 4,0 & 2,4 \\
\hline Plasmócitos & $0,80( \pm 0,60)$ & 0,2 & 0 & 0,2 & 0,2 \\
\hline Monócitos & $0,77( \pm 0,51)$ & 0 & 0 & 0 & 0,2 \\
\hline Macrófagos & $0,06( \pm 0,10)$ & 0 & 0 & 0,2 & 0 \\
\hline Células não classificadas & $0,49( \pm 0,28)$ & 0 & 0 & 0 & 0 \\
\hline Células degeneradas & $6,31( \pm 3,32)$ & 0,6 & 1,4 & 1,2 & 0,8 \\
\hline Células em mitose & $0,20( \pm 0,26)$ & 0 & 0,4 & 0,2 & 0,4 \\
\hline Total (outras) & $25,40( \pm 4,75)$ & 3,8 & 6,8 & 6,2 & 4,4 \\
\hline
\end{tabular}

ção produzir NADH (nicotinamida-adenina-dinucleotídio), um nucleotídio utilizado pela enzima metemoglobina-redutase na redução da metemoglobina diariamente formada, que é em torno de 3\%. A via pentose fostato produz NADPH (fosfato de nicotinamida-adenina-dinucleotídio), uma versão fosfatada do mesmo nucleotídio já descrito, que tem a função de atuar na redução do glutation oxidado, que é o principal responsável por manter a hemoglobina em uma forma estável. A inibição dessas vias pelo n-propil dissulfito causa depleção de NADH e NADPH, levando a quebra do mecanismo antioxidativo e conseqüentemente transformação de hemoglobina em metemoglobina (Harvey 1989, Fighera 2001).

Os achados clínicos da forma um pouco mais crônica dessa toxicose foram semelhantes aos descritos na literatura (Weiser 1992). Os sinais consistiam de palidez das mucosas, taquicardia e taquipnéia, evidenciando o processo anêmico que ocorre em consequiência da precipitação dos corpúsculos de Heinz (Desnoyers 2000). A formação de metemoglobina dentro do eritrócito causa agregação protéica e a desnaturação desse agregado na membrana da célula forma uma estrutura semelhante a uma bolha, conhecida como corpúscu- lo de Heinz (Beritic 1965). Essas inclusões são reconhecidas pelo sistema fagocítico mononuclear e os eritrócitos passam a ser retirados da circulação principalmente pelo baço e pelo fígado, fenômeno conhecido como hemólise extravascular. Ocorre também a opsonização do corpúsculo por IgG e complemento, mas de uma maneira menos eficiente, o que explica a razão da ruptura de alguns eritrócitos na circulação (hemólise intravascular) e da metemoglobinúria (Fighera 2001).

Os achados hematológicos dos quatro animais avaliados refletem um processo hemolítico, predominantemente extravascular, demonstrado pela grave anemia macrocítica hipocrômica, pela intensa regeneração e pela queda da relação mielóide:eritróide vista na medula óssea. A policromasia indica a liberação de grande quantidade de reticulócitos para a corrente circulatória, que em colorações de rotina são denominados policromatófilos, vindo daí o termo policromasia (Perrotta \& Finch 1972, Bain 1997). A anisocitose é reflexo do contraste entre os policromatófilos novos e grandes e os já menores eritrócitos lesados (Weiser 1992). A metarrubricitemia é resultante da hematopoese extramedular que foi confirmada pela histopatologia (Fighera 2001). A presença dos corpúsculos de Howell-Jolly, que são restos de núcleo, indica a rápida atividade mitótica do tecido eritróide no interior da medula óssea, o que faz com que alguns fragmentos de DNA não sejam extruídos (Lund 2000). A positividade para os corpúsculos de Heinz vem consolidar definitivamente o mecanismo patogenético da hemólise e os excentrócitos ("células mordidas") refletem a retirada dos corpúsculos de Heinz por partes (Naoum 1997). A intensa leucocitose associada à normoblastemia é chamada de reação leucoeritroblástica, ocorre em várias condições hemolíticas e acreditase ser decorrente da intensa estimulação medular inespecífica (Latimer \& Meyer 1992).

A análise química da urina demonstrou positividade para sangue oculto, o que ocorre tanto em casos de metemoglobinúria como na hemoglobinúria, mioglobinúria e hematúria (Navarro 1996). A constatação de altos níveis de metemoglobina sérica associada a essa positividade para sangue oculto na urina comprova que a coloração amarronzada vista no exame físico é compatível com metemoglobinúria, confirmando que parte da hemólise é intravascular.

Os achados de necropsia, caracterizados basicamente por esplenomegalia, e a avaliação histopatológica do baço e do fígado demonstrando hemossiderose, são compatíveis com hemólise extravascular e auxiliaram ainda mais na confirmação do diagnóstico (Valli 1993, Kelly 1993). A necrose centrolobular vista no animal 3 é provavelmente oriunda da anemia acentuada decorrente da metemoglobinemia grave (MacLachlan \& Cullen 1998).

\section{REFERÊNCIAS}

Bain B.J. 1997. Células Sangüíneas: um Guia Prático. 2ª ed. Artes Médicas, Porto Alegre. 334p.

Beritic T. 1965. Studies on Schmauch bodies. The incidence in normal cats (Felis domestica) and the morphologic relationship to Heinz bodies. Blood 25: 999-1008. 
Boothe D.M. 1990. Drug therapy in cats: mechanisms and avoidance of adverse drug reactions. J. Am. Vet. Med. Assoc. 196: 1297-1305.

Desnoyers M. 2000. Anemias associated with Heinz bodies, p. 178-184. In: Feldman B.F., Zinkl J.G. \& Jain N.C. (ed.) Schalm's Veterinary Hematology. 5th ed. Lippincott Williams \& Wilkins, Philadelphia. 1344p.

Farkas M.C. \& Farkas J.N. 1974. Hemolytic anemia due to ingestion of onions in a dog. J. Am. Anim. Med. Assoc. 10: 65-66.

Fighera R.A. 2001. Anemia em Medicina Veterinária. O Autor, Santa Maria. 217 p.

Gruhzit O.N. 1931. Anemia in dogs produced by feeding of whole onions and of onion fractions. Am. J. Med. Sci. 181: 812-814.

Harvey J.W. \& Rackear D. 1985. Experimental onion-induced hemolytic anemia in dogs. Vet. Pathol. 22: 387-392.

Harvey J.W. 1989. Erythrocyte metabolism, p. 185-273. In: Kaneko J.J. (ed.) Clinical Biochemistry of Domestic Animals. 4th ed. Academic Press, San Diego. 932p.

Harvey J.W. 2001. Atlas of Veterinary Hematology: Blood and Bone Marrow of Domestic Animals. W.B. Saunders, Philadelphia. 228p.

Hutchinsom T.W.S. 1977. Onion as a cause of Heinz body anemia and death in cattle. Can. Vet. J. 18: 358-360.

Jain N.C. 1986. Schalm's Veterinary Hematology. 4th ed. Lea \& Febiger, Philadelphia. 1221p.

Jain N.C. 1993. Essentials of Veterinary Hematology. Lea \& Febiger, Philadelphia. 417p.

Kaplan A.J. 1995. Onion powder in baby food may induce anemia in cats. J. Am. Vet. Med. Assoc. 207: 1405.

Kelly W.R. 1993. The liver and biliary system, p. 319-406. In: Jubb K.V.F., Kennedy P.C. \& Palmer N. (ed.) Pathology of Domestic Animals. Vol. 2. 4th ed. Academic Press, San Diego. 747p.

Koger L.M. 1956. Onion poisoning in cattle. J. Am. Vet. Med. Assoc. 129: 75.

Latimer K.S. \& Meyer D.J. 1992. Os leucócitos na saúde e na moléstia, p. 2281-2327. In: Ettinger S.J. (ed.) Tratado de Medicina Interna Veterinária. Vol. 4. $3^{\mathfrak{a}}$ ed. Manole, São Paulo. 2557p.
Lund J.E. 2000. Toxicologic effects on blood and bone marrow, p. 44-50. In: Feldman B.F., Zinkl J.G. \& Jain N.C. (ed.) Schalm's Veterinary Hematology. 5th ed. Lippincott Williams \& Wilkins, Philadelphia. 1344p.

MacLachlan N.J. \& Cullen J.M. 1998. Fígado, sistema biliar e pâncreas exócrino, p. 95-131. In: Carlton W.W. \& McGavin M.D. (ed.) Patologia Veterinária Especial de Thomson. $2^{\mathrm{a}}$ ed. Artes Médicas, Porto Alegre. 672p.

Naoum P.C. 1997. Hemoglobinopatias e Talassemias. Sarvier, São Paulo. 171p. Navarro C.E.K.G. 1996. Manual de Urinálise Veterinária. Varela, São Paulo. 95p.

Perrotta A.L. \& Finch C.A. 1972. The polychromatophilic erythrocyte. Am. J. Clin. Pathol. 57: 471-480.

Robertson, J.E., Christopher, M.M. \& Rogers, Q.R. 1998. Heinz body formation in cats fed baby food containing onion powder. J. Am. Vet. Med. Assoc. 212: 1260-1266.

Searcy G.P. 1998. Sistema hematopoético, p. 305-352. In: Carlton W.W. \& McGavin M.D. (ed.) Patologia Veterinária Especial de Thomson. $2^{a}$ ed. Artes Médicas, Porto Alegre. 672p.

Thorp F. \& Harshfield G.S. 1939. Onion poisoning of horses. J. Am. Vet. Med. Assoc. 94: 52-53.

Vallada E.P. 1999. Manual de Técnicas Hematológicas. Atheneu, Rio de Janeiro. $423 p$.

Valli V.E.O. 1993. The hematopoetic system, p. 101-266. In: Jubb K.V.F., Kennedy P.C. \& Palmer N. (ed.) Pathology of Domestic Animals. Vol. 3. 4th ed. Academic Press, San Diego. 653p.

Van Kampen K.R., James L.F. \& Johnson M.S. 1970. Hemolytic anemia in sheep fed wild onion (Allium validum). J. Am. Vet. Med. Assoc. 156: 328-332.

Weiser M.G. 1992. Os eritrócitos e os distúrbios associados, p. 2243-2280. In: Ettinger S.J. (ed.) Tratado de Medicina Interna Veterinária. Vol. 4. $3^{\underline{a}}$ ed. Manole, São Paulo. 2557p.

Weiser M.G. 1994. Disorders of erythocytes and erythropoiesis, p. 691-720. In: Sherding R.G. (ed.) The Cats Disease and Clinical Management. $2^{\mathrm{a}} \mathrm{ed}$. W.B. Saunders Company, Philadelphia. 2046p.

Williams H.H. 1941. Biochemical studies of the blood the dogs with n-propyl dissulfide anemia. J. Lab. Clin. Med. 26: 996-1008. 